\title{
A New Control Method of 2500KW Commutating Asynchronous Generator
}

\author{
Luan $\mathrm{Ru}^{\mathrm{a}}$, Jiang Zhijian ${ }^{\mathrm{b}}$ \\ No.1 Zhanlanguan Road, Xicheng District, Beijing Institute of Civil Engineering and Architecture, \\ China \\ aluanru@bucea.edu.cn, bjzjmail@bucea.edu.cn
}

Keywords: Integration; electrical machines; evaporative-cooling; power devices

\begin{abstract}
The 3/3 Phase Double-winding Induction Generator is designed for the high speed of the primal rotor. It is 2500 commutating asynchronous generator. An exciting winding is designed in the stator, and the control scheme is implemented by a static exciting device. In this paper the circuit principle is analyzed, also the control scheme is discussed. The simulation experiments are proposed and finished in this paper too.
\end{abstract}

\section{Introduction}

$2500 \mathrm{KW}$ commutating asynchronous generator is a new kind of high speed commutating generator with big power density and large power. It can realize the direct connection between the primal rotor and itself. As a result it can eliminate some damage factors such as huge volume and loud noise made by itself. Therefore the new commutating asynchronous generator can enhance the whole reliabilities of a power supply system ${ }^{[1]}$.

Because this generator will work at a very high speed (it will be 6000r/min), originally there are two projects to be chosen. One is the asynchronous project another is an isochronous one. Comparing with an isochronous one ${ }^{[6]}$, the asynchronous project has below advantages: A、The power density of the generator is higher so as to remarkably decrease its volume. $\mathrm{B}$ 、 The structure is very simple and the maintenance is also facility. So it will be economy. C、 The mechanism intensity of the whole generator will be very good.

But if a generator adopts asynchronous project, its control system will be very difficult to be designed and further to be realized. Once the control effect isn't ideal the performance of the asynchronous project will be disqualification ${ }^{[2]}$. So the key technology for the commutating asynchronous generator is the control system.

\section{Basic analysis method of a traditional asynchronous generator}

The asynchronous generator will be run in an independent power supply system. It usually connects capacitance paralleling with its output to realize the excitation. As we know, the process of building a voltage for asynchronous generating is mainly gradually raising the value of voltage by the magnetic induction that remains in the iron and the self-excitation through the parallel capacitance. The output voltage of the asynchronous generator is determined by some relationships among the rotor speed, the frequent, the exciting reactance and the inducting electromotive force, so we obtain the expression:

$$
U_{s}=f\left(X_{m}, \omega_{1}\right) \text {. }
$$

where the $X_{m}$ is exciting reactance and $\omega_{1}$ is angle frequency

From this expression, we can see that to traditional asynchronous generator when the rotor speed or the exciting capacitance is changed, the output voltage will change accordingly and sometime will fall to be zero. As a result the output for a traditional asynchronous generator is not steady very well. 
Combining with the independent power supply system, there are two schemes for the control of the asynchronous generator.

\section{A. Keep the output unchanging by control of the speed of the driving equipment}

There are also two methods to realize this scheme. One method is that the voltage frequency is controlled unchanging while the speed of the primal rotor is adjusted to satisfy the active power of the load. Meanwhile the compensation for reactive power should be supplied to keep the output voltage unchanging. Another one is that only the speed of the primal rotor is adjusted to supply the active power the load needed while keeping the output voltage unchanging. But the voltage frequency is not controlled at all, and any equipment for compensation is not needed either. These two methods are both established on the accurate control of the speed of the primal rotor in theories. Nevertheless the process of adjusting speed of the primal rotor is much more slower than the electric change of the generator. So in definite range to realize the accurate and fast control of the speed is too difficult to be designed.

B. Keep the output voltage unchanging by changing frequency meanwhile supplying the compensation for reactive power.

Now that the speed of the primal rotor can't be adjusted accurately, the only method that keeping the output voltage of the asynchronous generator constant is to adjust the working frequency in real time according to the speed of the primal rotor. So this paper chooses adjustable frequency driving to keep the output voltage invariable. But the method is very difficult to realized and controlled.

To any load, if the sliding power is too big, the output voltage of the generator will rise greatly, otherwise it will decrease distinctly. In addition the sliding power will influence the celerity vand the reliability 、 and the stability of a whole power supply system[4]. Considering all the various factors this paper offers a new kind of asynchronous generating project, which the power is generated by inducing two sets of stator winding. Therefore the $2500 \mathrm{KW}$ commutating asynchronous generator has two sets of stator winding. One of them is main power winding which generates three phase AC and supply direct current to loads by its inside rectifier. While another winding is still three phase ac and regarded as compensation. It can supply the lost exciting magnet flux caused by the load. As a result the two sets of winding can keep the output dc voltage of the rectifier[3] unchanging.

As the two sets of stator winding have the same number of pole, it is apparent that the frequencies of them are the same too. So when the voltage frequency of the compensating winding is kept constant, the voltage frequency of the power winding is also certainly kept the same. Accordingly the $2500 \mathrm{KW}$ commutating asynchronous generator can adjust the working frequency, and keeps it steady. The adjusting process is described below. When the load of generator is void, here voltage is built by port capacitance. And when the generator is working from void load to full load, the input voltage frequency and magnitude of the compensating winding is adjusted by a controller to supply the needed excitation for the main power winding, so as to keep the output voltage unchanging. Further more when the generator is well-balanced, the compensating winding mainly is used to supply reactive power needed by the whole system compensating exciting magnet flux. But the active power existed in the compensating winding is very small comparatively. The whole structure control principle is shown in figure 1.

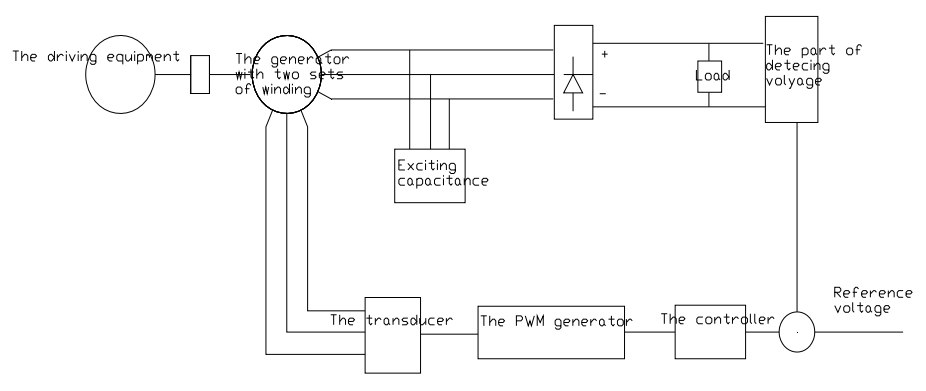

Fig.1. The sketch map of the new control structure 
In order to testify the control feasibility of the two sets of stator winding, we rebuild a common three phase asynchronous motor through designing its stator newly. It is that the original stator winding is changed into two sets of three-phase winding which are different in some degree in their electric angles. Before analyzing we have made some reasonable hypotheses: A、 Ignore the loss of the transducer; $\mathrm{B}$ 、 The speed of the driving equipment is constant.

The basic control way is described below.

A transducer is located at one side of the compensating winding. Its modulating rate is fixed, but its output frequency will be determined by the dc voltage of the rectifier belonging to the main power winding. When the actual dc voltage is bigger than the set value the working frequency will be increased to decrease the absolute value of the sliding power. Otherwise the working frequency will be decreased, until the dc voltage of the rectifier is equal to its set value[4][5]. In theory there are two projects to realize the control of working frequency. One project is that the transducer is separated from the dc side of the rectifier. The control structure of the project is shown in Figure 2.

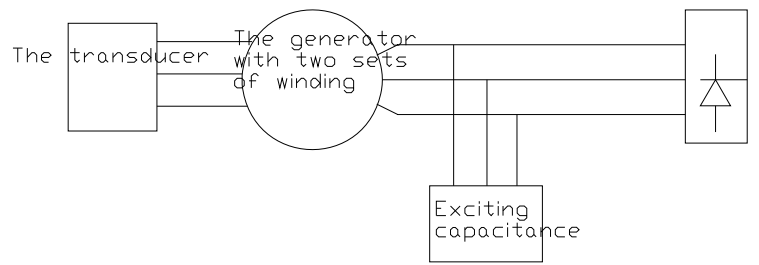

Fig. 2. The sketch map of the transducer separating from the dc side of the rectifier

When the system is running, to each steady state once the speed is fixed the frequency is also fixed. We can conclude that in the ac side of the transducer the current is a pure reactive component compared with the voltage. Thus we can regard the transducer as capacitance which can be adjusted by itself according to the actual running state of the generator. So the transducer sometime works as an inverter and sometime works as a rectifier, while the power supplied by the input primal rotor is exported through the main power winding of the generator. It is apparent that output dc voltage of the commutating asynchronous generator can be steady by adjusting the working frequency. Another project is that the transducer is connected to the dc side of the rectifier. But this project is so instable as to be denial by this paper.

In order to validate the control principle described above, we have made an experimental model and on it we have made some loading experiments. The key parameters of the commutating asynchronous generator model are that its number of pole pair is 2, and the voltages of its two sets of winding are both $220 \mathrm{~V}$, and the current of the main power winding is $10.6 \mathrm{~A}$, while the current of the compensation winding is 5.3A. The capacitance of dc side is $1650 \mu \mathrm{F}$. And the transducer is IPM model which is PM75CVA120. The exciting capacitance is chosen $100 \mu \mathrm{F}$ and $20 \mu \mathrm{F}$ respectively to do experiments. The experimental results is given and explained below.

When the exciting capacitance is chosen $100 \mu \mathrm{F}$ and $20 \mu \mathrm{F}$ respectively, the corresponding absolute value of the slid angle frequency is no more than 0.02 . During the process we sampled the value of the dc current and voltage of the generator and other key data as shown in figure 4-figure6. In these figures the y-axis is original sampling data while there are 400 sampling times in a fundamental sampling period. From the sampling waveform the phase angle deference between the current and voltage can be estimated. And it shows that the phase current leads the phase voltage nearly 90 degree, which testifies that the ac side of the transducer is reactive. In figure 4 when the load current is increasing, the lost exciting magnet flux is increasing too, but the current of compensating winding is also added to supply the lost magnet flux. And the bigger the capacitance is the smaller the compensating current is needed. In addition we finished the load experiment under suddenly adding load condition. The voltage is still kept the same as the set value at last. 


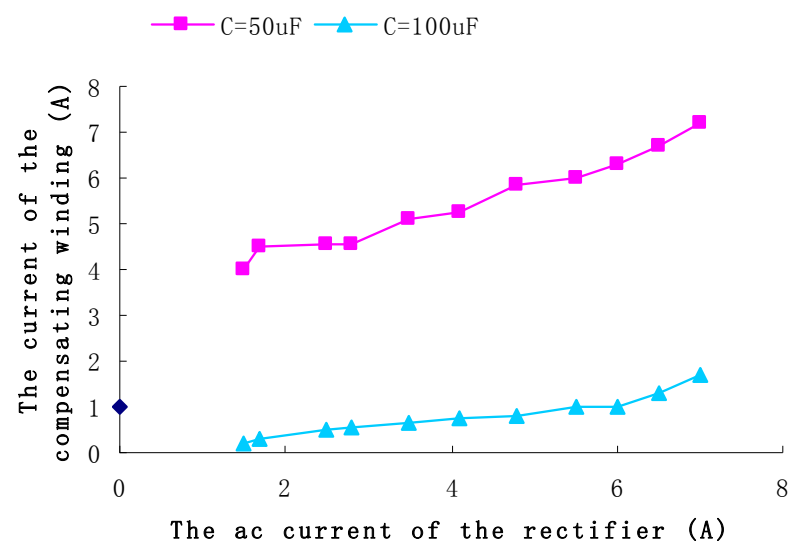

Fig.4. The relationship between the load and compensating winding currrent

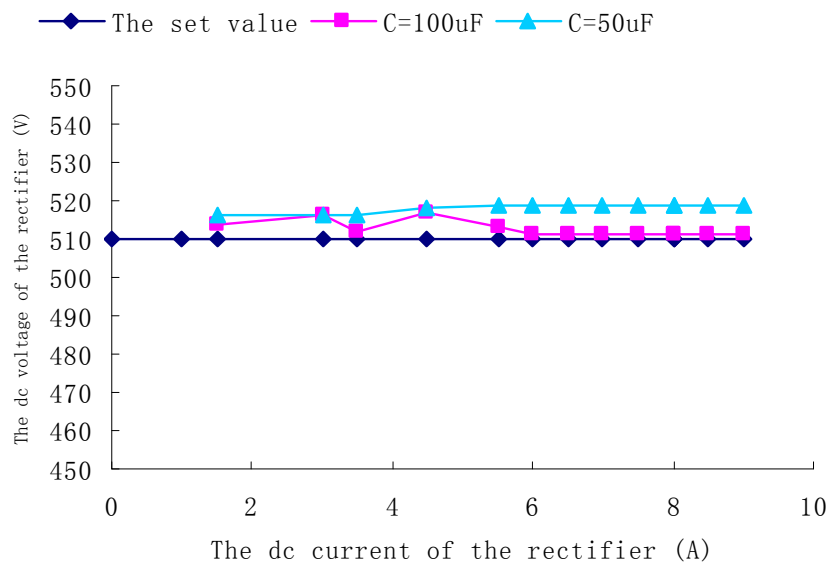

Fig.5. The relationship between the load and the output dc voltage

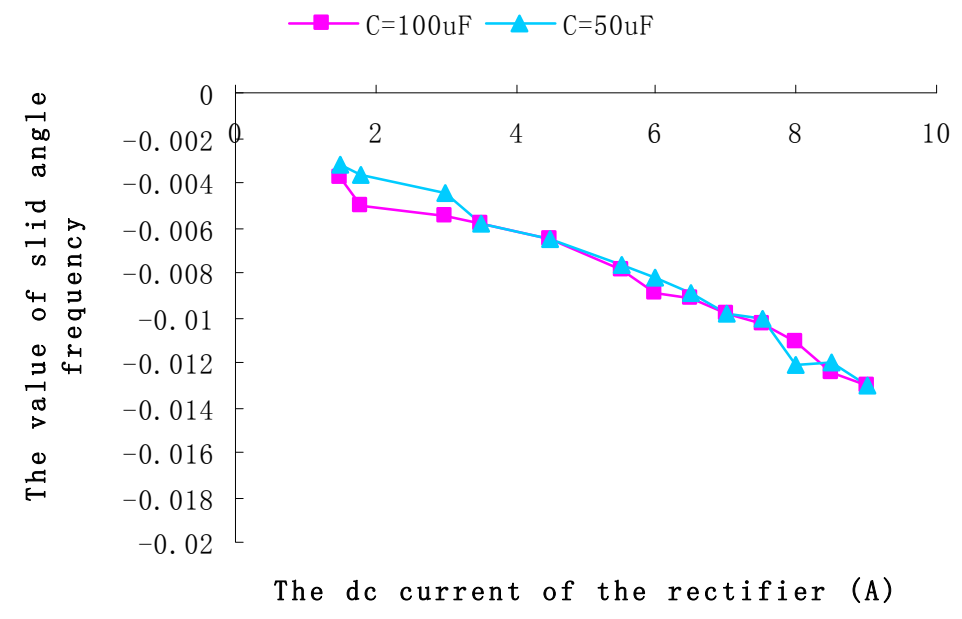

Fig.6. The relationship between the load and the slid angle frequency

\section{Conclusion}

A new kind of $2500 \mathrm{KW}$ commutating asynchronous generator is offered by this paper. In order to control effectively the output voltage to be constant, a new design project of two sets of stator winding is brought forward. The two sets winding will cooperate each other. While one of them is the main power winding to supply a constant dc voltage to loads, another is the compensating winding to control the working frequency to keep the output voltage unchanged. The whole control process has been analyzed and validated to be feasible by a serious of theory and some experiments.

\section{References}

[1] Xiao Fei, Zhang Botao, Ma Weiming el al. "Dual stator winding generator and the excitation 
control ” Automation of Electric Power System, vol. 27, pp: 26-29, August 2003

[2] Wang Dong, Ma Weiming, Xiao Fei, . el al "Research on dual stator winding generator with static excitation regulator” Proceeding of the CSEE, vol. 23, pp: 145-150, May 2003

[3] Zargari N R, Joos G. “ Performance investigation of a current-controlled voltage-regulated PWM rectifier in rotating and stationary frames” IEEE Trans Industrial Electronics, vol. 42, pp: 396-401, April 1995

[4] Fang Zheng Peng, John W McKeever. “ A power line conditioner using cascade multilevel inverters” IEEE Trans on Industrial Application, vol. 34, pp: 1293-1298, July 1998

[5] Fang Zheng Peng, Jih-sheng LAI. “ Dynamic performance and control of a static var generator using cascade multilevel inverters” IEEE Trans on Industrial Application, vol. 33, pp: 1009-1015, March 1997

[6] Zhang Xiaofeng Stability of synchronous generator-rectifier-load system, Doctoral Dissertation. Beijing: Tsinghua University, 1995 